\title{
Phenotypic and genetic characterization of a novel phenotype in pigs characterized by juvenile hairlessness and age dependent emphysema
}

\author{
Camilla S Bruun ${ }^{1}$, Claus B Jørgensen ${ }^{1}$, Lene Bay¹, Susanna Cirera1, \\ Henrik E Jensen ${ }^{2}$, Páll S Leifsson ${ }^{2}$, Jens Nielsen ${ }^{3}$, Knud Christensen ${ }^{1}$ and \\ Merete Fredholm*1
}

Address: ${ }^{1}$ Department of Basic Animal and Veterinary Sciences, Faculty of Life Sciences, University of Copenhagen, Grønnegårdsvej 3, DK-1870 Frederiksberg, Denmark, ²Department of Veterinary Pathobiology, Faculty of Life Sciences, University of Copenhagen, Ridebanevej 3, DK-1870 Frederiksberg, Denmark and ${ }^{3}$ National Veterinary Institute, Technical University of Denmark, Lindholm, DK-4771 Kalvehave, Denmark

Email: Camilla S Bruun - cvb@life.ku.dk; Claus B Jørgensen - chj@life.ku.dk; Lene Bay - lene_bay@hotmail.com;

Susanna Cirera - scs@life.ku.dk; Henrik E Jensen - helj@life.ku.dk; Páll S Leifsson - ple@life.ku.dk; Jens Nielsen - jn@vet.dtu.dk;

Knud Christensen - kc@life.ku.dk; Merete Fredholm* - mf@life.ku.dk

* Corresponding author

Published: 12 June 2008

BMC Genomics 2008, 9:283 doi:10.1 186/147|-2164-9-283

This article is available from: http://www.biomedcentral.com/l47I-2164/9/283

(C) 2008 Bruun et al; licensee BioMed Central Ltd.

This is an Open Access article distributed under the terms of the Creative Commons Attribution License (http://creativecommons.org/licenses/by/2.0), which permits unrestricted use, distribution, and reproduction in any medium, provided the original work is properly cited.
Received: 28 November 2007

Accepted: 12 June 2008

\begin{abstract}
Background: A pig phenotype characterized by juvenile hairlessness, thin skin and age dependent lung emphysema has been discovered in a Danish pig herd. The trait shows autosomal co-dominant inheritance with all three genotypes distinguishable. Since the phenotype shows resemblance to the integrin $\beta_{6}-/-$ knockout phenotype seen in mice, the two genes encoding the two subunits of integrin $\alpha_{v} \beta_{6}$, i.e. ITGB6 and ITGAV, were considered candidate genes for this trait.

Results: The mutated pig phenotype is characterized by hairlessness until puberty, thin skin with few hair follicles and absence of musculi arrectores pili, and at puberty or later localized areas of emphysema are seen in the lungs. Comparative mapping predicted that the porcine ITGB6 andITGAV orthologs map to SSCI5. In an experimental family $(n=\mid 13)$, showing segregation of the trait, the candidate region was confirmed by linkage analysis with four microsatellite markers. Mapping of the porcine ITGB6 and ITGAV in the IMpRH radiation hybrid panel confirmed the comparative mapping information. Sequencing of the ITGB6 and ITGAV coding sequences from affected and normal pigs revealed no evidence of a causative mutation, but alternative splicing of the ITGB6 pre-mRNA was detected. For both ITGB6 and ITGAV quantitative PCR revealed no significant difference in the expression levels in normal and affected animals. In a western blot, ITGB6 was detected in lung protein samples of all three genotypes. This result was supported by flow cytometric analyses which showed comparable reactions of kidney cells from affected and normal pigs with an integrin $\alpha_{v} \beta_{6}$ monoclonal antibody. Also, immunohistochemical staining of lung tissue with an integrin $\beta_{6}$ antibody showed immunoreaction in both normal and affected pigs.
\end{abstract}

Conclusion: A phenotype resembling the integrin $\beta_{6}-/$ - knockout phenotype seen in mice has been characterized in the pig. The candidate region on SSCI5 has been confirmed by linkage analysis but molecular and functional analyses have excluded that the mutated phenotype is caused by structural mutations in or ablation of any of the two candidate genes. 


\section{Background}

A phenotype, characterized by juvenile hairlessness, thin skin and age dependent lung emphysema, has been encountered in a Danish pig herd. All piglets showing the trait are descendants of a specific boar and the trait shows autosomal co-dominant inheritance. At the age of six months hair growth is increased but not fully restored. A similar phenotype has been described in knock-out mice deficient of the ITGB6 gene (ITGB6-/-) encoding the integrin $\beta_{6}$-subunit. These mice display juvenile hairlessness mainly on their neck, head and inner thighs, macrophage infiltration of the dermis and lymphocyte infiltration of the conductive airways of the lung. At the age of puberty, hair growth is resumed [1] and later, age dependent lung emphysema appears [2].

Integrins are a family of cell surface heterodimers, each consisting of an $\alpha$ - and a $\beta$-subunit. The integrin molecules are anchored in the cell membrane where they mediate a wide range of cell-cell and cell-matrix interactions [3]. The $\beta_{6}$-subunit pairs only with the $\alpha_{\mathrm{v}}$-subunit constituting integrin $\alpha_{\mathrm{v}} \beta_{6}[4]$. The $\alpha_{\mathrm{v}}$-subunit, however, pairs with five different $\beta$-subunits [5] and plays a central role with respect to normal embryonic development and survival. Ablation of ITGAV in mice causes malformations and embryonic or postnatal death [6].

The expression of $\alpha_{v} \beta_{6}$ is restricted to epithelia and seems predominant in various developing organs of the embryo/ fetus $[7,8]$. Besides, various patho-biological events induce a neo-expression of this integrin, e.g. tumor genesis $[7,9]$, wound healing [10], and general cases of clinical or subclinical inflammation [7].

The resemblance between the phenotype detected in the pigs and the phenotype of the ITGB6-/- mice suggests ITGB 6 as a candidate gene for the mutated pig phenotype. However, since the ITGB6 polypeptide dimerizes with ITGAV the gene encoding ITGAV is also considered a possible candidate.

In this paper the mutated pig phenotype is characterized macroscopically and histologically. Furthermore, taking the outset in linkage studies, sequencing and functional studies, ITGAV and ITGBG are evaluated as candidates for the phenotype.

\section{Results}

\section{Phenotype 0-6 months}

Based on the founder boar and his offspring an experimental population $(\mathrm{n}=113)$ was established. A clear difference in amount of hair was observed in the pedigree, i.e. animals could be scored as normal (hh), heterozygous (Hh) and homozygous (HH) according to these observations. Hh pigs displayed hairless areas primarily on the

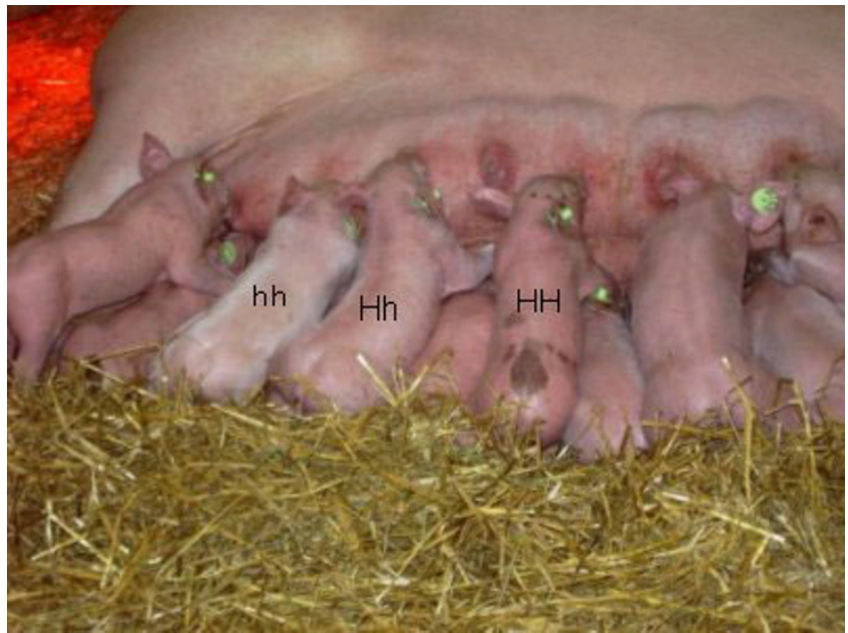

Figure I

Juvenile hairlessness in pigs. All three genotypes $(\mathrm{HH}, \mathrm{Hh}$ and hh) represented in one litter.

back and rear, whereas HH pigs were almost entirely hairless. The degree of hairlessness varied somewhat within each genotype group. The skin appeared thin and reddish, mainly in the $\mathrm{HH}$ pigs but also in the Hh pigs (see Figure $1)$.

Shoulder and thigh skin samples from nine two months old pigs (three of each genotype) were evaluated histologically. The thickness of the dermis was measured and the hair follicles were counted. No lesions were observed apart from a few small areas with erosions and mild hyperkeratosis, which are normally present in the skin of piglets. However, in skin sections from both shoulder and thigh the dermis was significantly thinner in the $\mathrm{HH}$ and Hh groups compared to the hh group $(\mathrm{p}=0.0001)$. There was no significant difference between the $\mathrm{HH}$ and $\mathrm{Hh}$ groups. The HH group had significantly fewer hairs/hair follicles than the Hh group $(\mathrm{p}=0.0001)$, and the $\mathrm{Hh}$ group had significantly fewer hairs/hair follicles than the hh group $(\mathrm{p}=0.01)$.

Musculi arrectores pili were totally absent in skin from $\mathrm{HH}$ animals and only rarely detected in skin from Hh animals. Another morphologic difference was the tissue surrounding the sweat glands in the skin. In all skin sections these glands were found within the dermis close to the subcutis, but in HH skin they were embedded in connective tissue and in hh skin they were surrounded by fat tissue (see Figure 2).

Histological examination of lung tissue samples from the same nine pigs showed signs of interstitial pneumonia to a variable extend characterized by thickening of the alveolar walls due to septal infiltrates of mononuclear cells and 

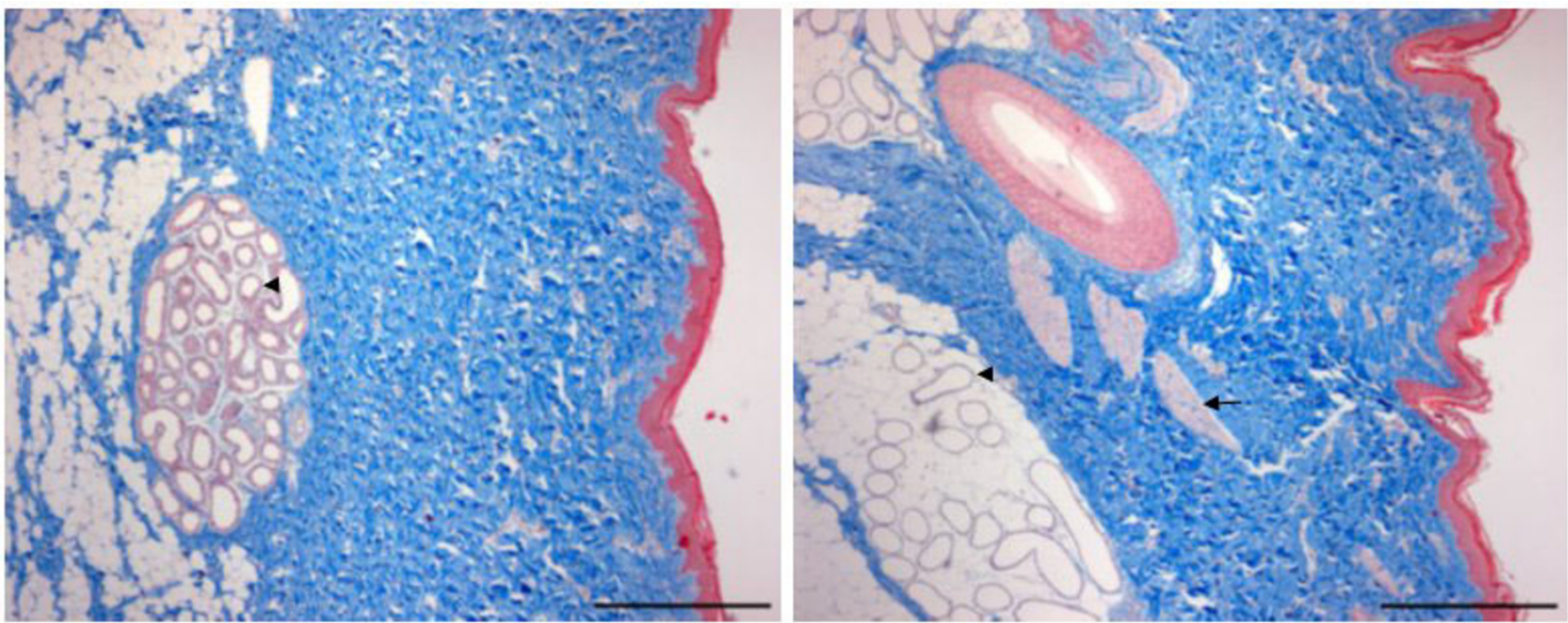

\section{Figure 2}

HH and hh skin sections. Skin from one HH (left) and one hh (right) pig, both two months old. The HH skin is characterized by a lack of hair/hairfollicles and musculi arrectores pili (arrow), thin dermis and sweat glands (arrowhead) embedded directly in the connective tissue (blue). Trichrome staining, bar $=200 \mu \mathrm{m}$.

hyperplasia of type II pneumocytes. Within some of the interstitial infiltrates syncytial cells were seen. Also hyperplasia of the bronchial associated lymphoid tissue (BALT) was present to a variable extend in all animals.

\section{Phenotype of adults}

Around the age of six months, hair growth was resumed but even in adult $\mathrm{HH}$ pigs the hair coat was very thin and frizzy. Hh animals grew slightly more hair; however they did not recover normal hair growth. The skin of both $\mathrm{HH}$ and Hh adults became very scaly and histological examination of skin sections revealed noticeable hyperkeratotic epidermis. Musculi arrectores pili were still absent and the sweat glands were also surrounded by connective tissue in the skin of adult $\mathrm{HH}$ animals.

Lung tissue of the HH animals ( $\mathrm{n}=5$, ages from 8 to 21 months) and older Hh animals ( $\mathrm{n}=3$, ages from 29 to 42 months) also showed interstitial pneumonia as well as areas with alveolar enlargement (localized areas of emphysema) (see Figure 3).

Lung tissues from one yonger adult Hh animal $(\mathrm{n}=1$, age of 12 months) and the hh animals $(n=4$, ages of 8 months) showed normal, diffusely distended alveolar lumina. Some septal enlargement was observed in the lung tissue from the Hh and one hh.

\section{Candidate region}

ITGB6 and ITGAV have been shown to be mapped to MMU2 at positions 34 and $46 \mathrm{cM}$ respectively and to the orthologous region on HSAP2 at positions 160.7 and 187.2 Mb respectively $[11,12]$. Based on a human-porcine comparative map the ITGB6 and ITGAV candidate region was identified on SSC15 [13].

\section{Radiation hybrid (RH) mapping}

The porcine ITGB6 and ITGAV genes were mapped by RH mapping. ITGB6 was mapped to the interval between the markers CL327053 and CL378417 [14] on SSC15 corresponding to an approximate position of $65 \mathrm{cM}$ [13]. ITGAV was mapped to the interval between the markers CL356064 and CL360872 corresponding to an approximate position of $71 \mathrm{cM}$ on SSC15 [13]. These results are in agreement with the orthologous positions of the human ITGBG and ITGAV.

\section{Linkage study}

A linkage study was performed with seven microsatellite markers selected from the candidate region on SSC15. The locus underlying the mutated phenotype was linked to four of the seven microsatellites: S0149, SW1945, KS155 and SW906 [15] with LOD scores from 8.4 to12.1. The mutated locus could be genetically mapped between markers S0149 and SW906 ( $25 \mathrm{cM}$ ) with high confidence (LOD > 3), however finer mapping with SW1945 and KS155 could not be supported by a LOD score higher than 3. Keeping the linear order of the markers from the published linkage map [15] the result can be illustrated as shown in Figure 4. 


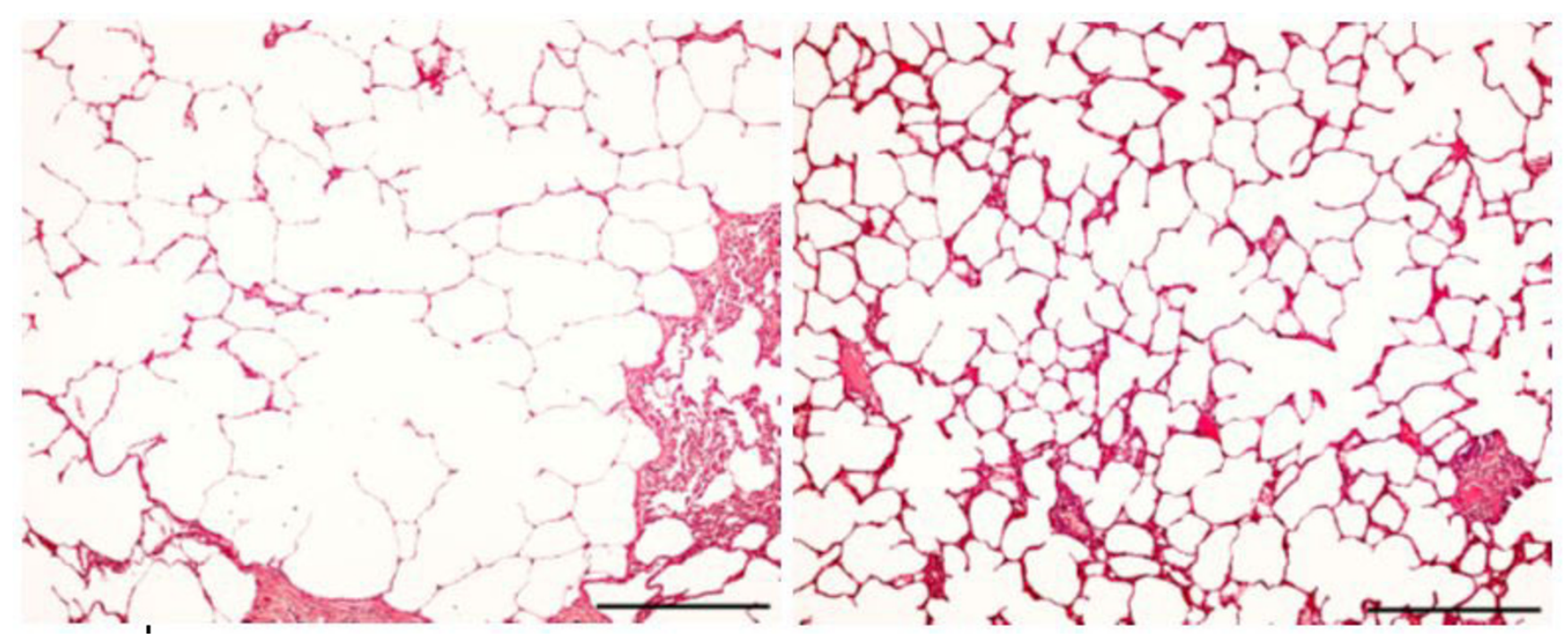

Figure 3

Age dependent lung emphysema. Lung from a HH boar of nine months, emphysema area (left) and normal area (right). Haematoxylin and eosin staining, bar $=200 \mu \mathrm{m}$.

\section{ITGB6 coding sequence}

ITGB6 specific PCR amplification from skin cDNA samples gave either little or no PCR products, whereas the transcript was easily amplified in lung cDNA. PCR products covering the whole ITGB6 transcript were cloned and sequenced. The porcine ITGB6 coding sequence [GenBank: AM114538] consists of 2367 nucleotides covering 15 exons. A short 3'UTR of only 16 nucleotides precedes the polyA tail. The amino acid sequence [Swiss-Prot: Q1RPR6] has a similarity of 98.5 and $95.4 \%$ respectively to the human [Swiss-Prot: P18564] and mouse [SwissProt: Q9Z0T9] ITGB6 amino acid sequences. It contains $\beta 6$ specific features like the 2 Arg-Gly-Asp (RGD) sequences in the extracellular part and the 11 amino acid extension of the carboxyl terminus [16].

As observed for many other integrins [17] sequence analysis revealed alternative splice forms of the pre-mRNA. The following alternative splicing events were observed: skipping of exon 2 leading to frameshift and generation of a stop codon in exon 3; skipping of exon 6 (in frame); skipping of exon 12 through 15 with the concomitant inclusion of an additional sequence [GenBank:AM114539] that does not show homology to any of the splice variants reported in other species. In addition, there was evidence of concomitant skipping of exons 13 and 14. All splice forms of ITGB6 were detected in both hh and HH pigs. Alignment of the HH and hh ITGB6 coding sequences revealed one synonymous SNP but no allelic variants of this SNP showed cosegregation with the phenotype.

\section{ITGAV coding sequence}

The porcine ITGAV coding sequence was generated by sequencing ITGAV specific PCR products amplified in lung cDNA. The porcine ITGAV coding sequence [GenBank: AM491473] consists of 3140 nucleotides and the 3' UTR consists of 831 nucleotides. The amino acid sequence [Swiss-Prot: A2RQD8] has a similarity of 95.8 and $94.2 \%$ respectively to the human [Swiss-Prot:

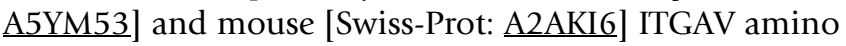
acid sequences. No SNP's were detected in the alignment of the HH and hh ITGAV sequences.

\section{Quantitative PCR}

Real time quantitative PCR (qPCR) was used to study expression of ITGB6 and ITGAV in animals with the hh and HH genotype respectively. In ITGB6, two different target sequences were included: one within exon 3 and one spanning exon 9 to 10 . These exons seem to be present in all detected splice variants and the two target sequences provide an opportunity to compare the expression of two different parts of the transcript. In ITGAV the target sequence was spanning exon 1 to 3 . The expression of ITGBG and ITGAV was analyzed using REST [18] and the pair wise fixed reallocation randomisation test included in the programme. All three target sequences showed a slight upregulation in the $\mathrm{HH}$ group but the differences did not reach the significance level ( $p>0.05$ ) (see Table $1)$.

\section{Western Blot}

Lung protein samples from one mouse (control) and three pigs representing the genotypes $\mathrm{hh}, \mathrm{Hh}$ and $\mathrm{HH}$ 


\section{$-F^{2}=$}

\section{Figure 4}

Linkage region on SSCI 5. The mutated locus has been mapped to a position between the markers SOI49 and SW906 on SSCI5.

were included in the western blot analysis. The detection was performed using an anti-mouse integrin $\beta_{6}$ antibody. We observed a band of approximately $98 \mathrm{kDa}$ in each of the pigs. These bands had an equal intensity and were comparable with the control mouse band of approximately $90 \mathrm{kDa}$ (see Figure 5).

\section{Flow cytometry}

The integrin $\alpha_{v} \beta_{6}$ expression on kidney cells was compared between the hh $(n=2)$ and HH $(n=2)$ genotypes by flow cytometry. This experiment was included to study the expression in a non-lung tissue. For the two HH pigs, approximately $85 \%$ of the kidney cells were integrin $\alpha_{v} \beta_{6}$ positive. For the hh pigs, the proportion of positive cells was lower, i.e. about $69 \%$, as illustrated in Figure 6 . The mean fluorescence intensity of positive cell populations, however, was at the same level for individual pigs (data not shown), showing a comparable integrin $\alpha_{\mathrm{v}} \beta_{6}$ expression of the two genotypes.

\section{Immunohistochemistry}

Immunohistochemistry was performed with goat antimouse integrin $\beta 6$ antibody on lung tissue from seven pigs representing young and adult animals of the three genotypes (hh, $\mathrm{Hh}$ and $\mathrm{HH}$ ). Lung tissues from all three genotypes stained with the antibody and the immunoreaction was localized to the alveolar, bronchiolar, bronchial and glandular epithelial cells (see Figure 7).

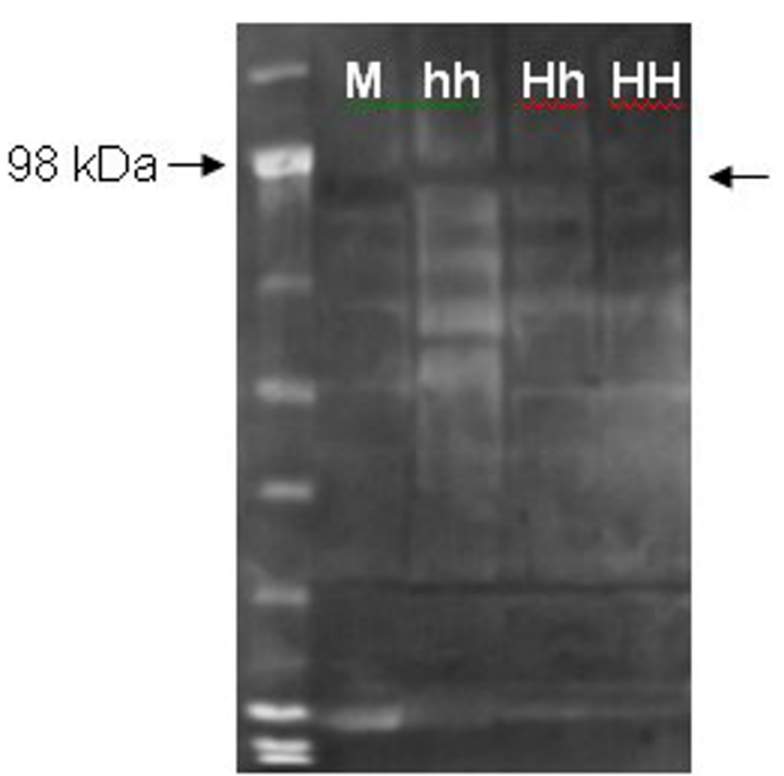

\section{Figure 5}

ITGB6 western blot. Western blot performed with lung protein from mouse $(M)$ and three pigs representing the three genotypes (hh, $\mathrm{Hh}$ and $\mathrm{HH}$ ). The left arrow points out the $98 \mathrm{kDa}$ band of the marker. The right arrow indicates the ITGB6 specific bands of the pigs with approximate sizes of 98 $\mathrm{kDa}$. The murine ITGB6 specific band has a comparable size of approximately $90 \mathrm{kDa}$.

\section{Discussion}

The mutated pig phenotype was originally detected in a boar and among his offspring. It is most probably caused by a spontaneous mutation in the founder boar and the corresponding phenotype has resemblance to that of the ITGB6-/- knockout mice. The only not coinciding features are the distribution of the hairlessness and the macrophage and lymphocyte infiltrations observed in the skin and lungs respectively of the knock-out mice. The interstitial pneumonia detected in the lungs of both young and adult pigs is most likely due to infection with porcine circovirus-2 (PCV-2) which is widespread within the Danish population of pigs [19] including our experimental population.

The fact that the hairlessness of the heterozygous pigs is intermediate indicates, that one functional copy of the gene in question is not enough to maintain a normal skin phenotype (or one dysfunctional copy can impair the "normal" pathway). The mutation seems to affect secondary structures of the skin in general. We have observed aplasia not only of hair follicles but also of musculi arrectores pili. Furthermore, the sweat glands are embedded directly in the connective tissue without any surrounding fat tissue. Also, the thickness of the dermis is affected. 
(A)

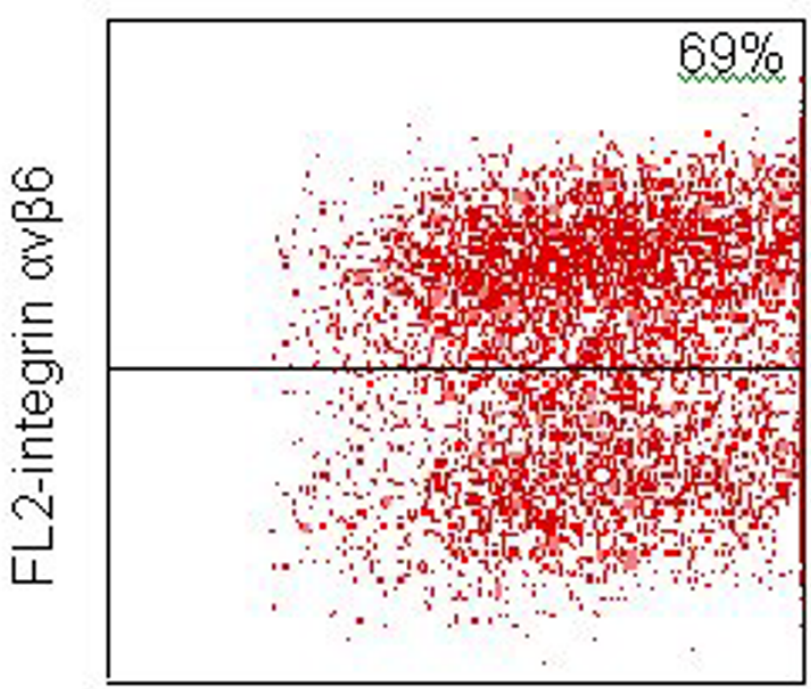

(B)

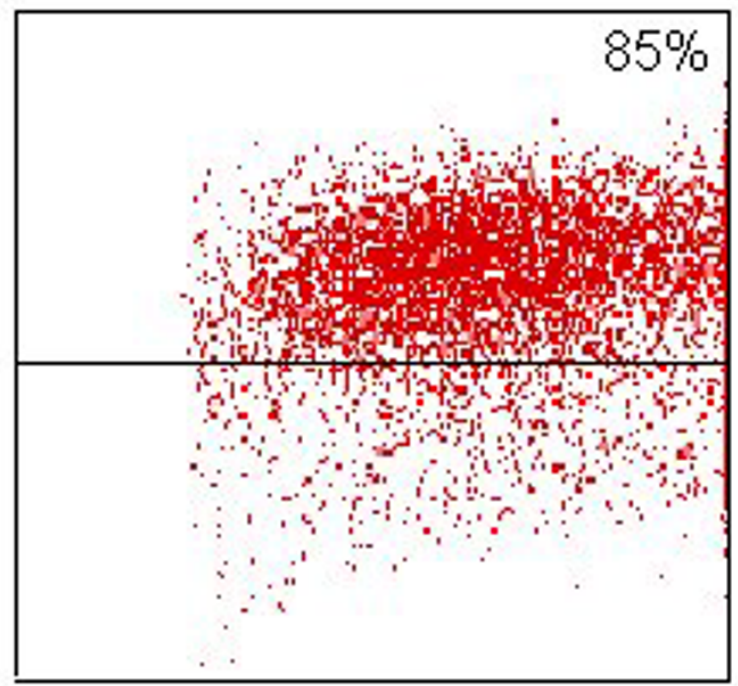

\section{Figure 6}

Integrin $\alpha \mathbf{v} \beta 6$ flow cytometry. SSC (sideward light scattering)/FL2 (fluorescence 2)-integrin av $\beta 6$ dot plots of primary kidney cells from 2 six-week-old pigs with genotypes hh $(A)$ and $H H(B)$, respectively. Dots in the upper right quadrant represent cells reactive with $\mathrm{mAb} 2077 \mathrm{Z}$, and the percentage of integrin av $\beta 6$ positive cells is indicated for each pig.
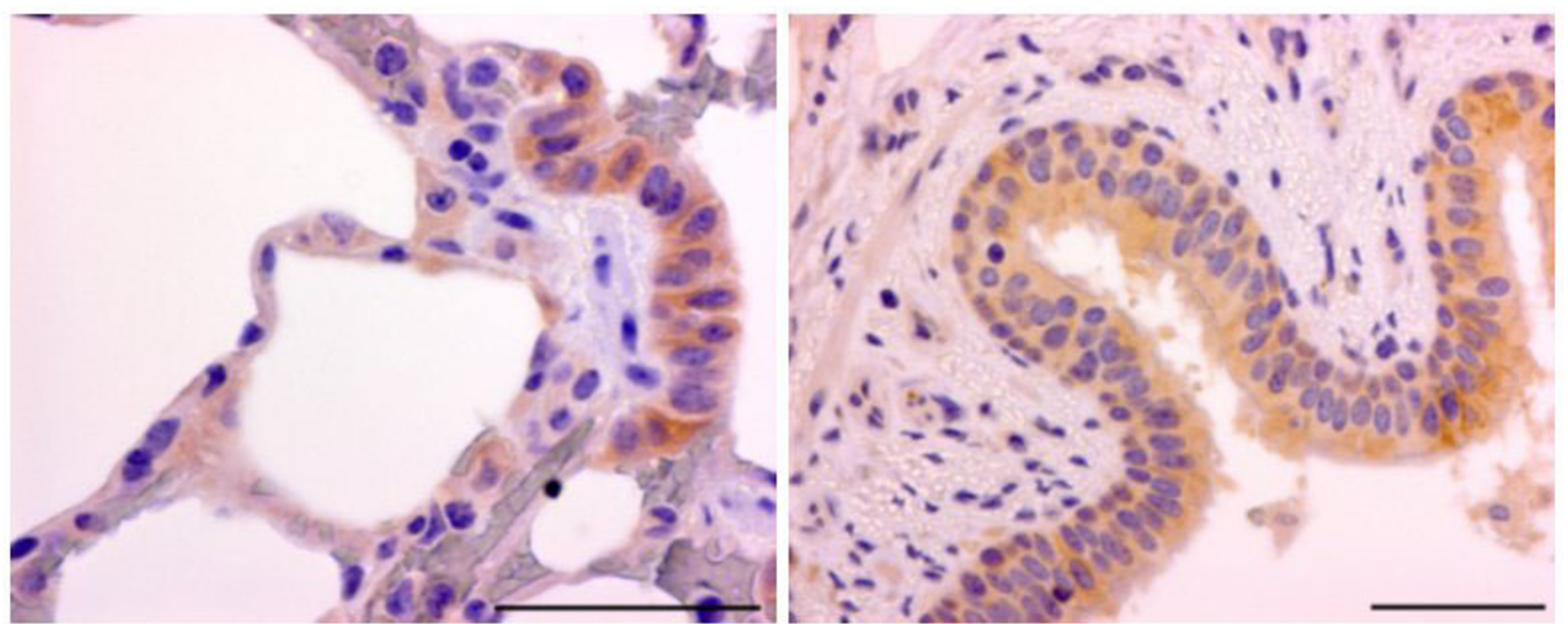

Figure 7

Integrin $\alpha \mathbf{v} \beta 6$ immunohistochemical staining of lung tissues. Lung tissues from all three genotypes stained with the integrin $\alpha v \beta 6$ antibody. Representative pictures are shown. The red-brown colour localized to the alveolar, bronchiolar, bronchial and glandular epithelial cells indicates immunoreaction. Bar $=50 \mu \mathrm{m}$.

Both heterozygous and homozygous pigs develop age dependent lung emphysema. Our animal material has not been sufficient to provide an "age of onset" of emphysema within the two genotype groups but our data suggest that emphysema appears around the age of puberty in homozygous animals and that the heterozygous pigs 
maintain a normal lung phenotype longer than the homozygous pigs.

Although the candidate region on SSC15 was confirmed, our results so far have not supported ITGB 6 as a candidate gene. First, we did not detect any non-synonymous mutations in the coding region of the ITGB6 gene in the $\mathrm{HH}$ animals. A difference in the expression level of ITGB6 in the two genotype groups could have pointed towards a mutation in the regulatory region of this gene. Even if the ITGB 6 expression was upregulated in the HH group, the difference was not significant. A reason for the upregulation could be the interstitial pneumonia observed in the $\mathrm{HH}$ group, as ITGB6 is known to be upregulated in response to inflammation $[2,7,20]$. In the hh group we observed interstitial pneumonia in only one pig. The western blot showed an apparently equal expression of the ITGB6 protein in the three groups.

The porcine gene encoding the integrin $\alpha_{\mathrm{v}}$ subunit (ITGAV) was mapped to a position on SSC15 within the candidate region. A mutation in ITGAV would be expected to have far more severe consequences, as the $\alpha_{\mathrm{v}}$ subunit pairs with five different $\beta$ subunits [5]. In mice, for instance, the ablation of ITGAV causes embryonic or postnatal death [21]. Still, a mutation in ITGAV could not be entirely excluded as such a mutation might reside in a region interacting specifically with the $\beta 6$ subunit. Sequencing of the ITGAV HH and hh coding sequences, however, did not reveal any potential causative mutation, and also, quantitative PCR results revealed an equal ITGAV expression in $\mathrm{HH}$ and hh pigs.

These observations were supported by the flow cytometric demonstration of high levels of the $\alpha_{v} \beta_{6}$ receptor on kidney cells from $\mathrm{HH}$ animals and immunohistochemical detection of the receptor in HH lung sections. The presence of the receptor in $\mathrm{HH}$ pigs was furthermore confirmed by immunocytochemical staining of monolayer HH pig kidney cells (data not shown).

The pathogenesis underlying the hairlessness in the knockout mice is so far unknown. Huang et al. [1] observe fewer hair follicles and numerous degenerating hair follicles surrounded by mononuclear cells in the hairless dermis of the ITGB6-/- mice, indicating an inflammatory response. It has been demonstrated that the expression of ITGB6 is upregulated in response to injury and/or inflammation and that integrin $\alpha_{\mathrm{v}} \beta_{6}$ exerts a modulating effect on the inflammatory response $[2,7,20]$. Huang et al. [1] argue that the lack of integrin $\alpha_{v} \beta_{6}$ in ITGB6-/- mice allows for minor skin irritations to cause an escalated inflammatory response resulting in hairlessness, for instance in the neck skin where the mother lifts her pups. Hair loss due to skin irritation can however, be excluded in the pig since the phenotype is inborn and without any sign of inflammatory response. Interestingly, our more detailed histological characterization of the skin show that hair follicles are present at a low frequency, however, without presence of musculi arrectores pili. This indicates that the mechanism underlying the phenotype has a subtle influence on cell migration/cellular development during embryonal development.

In contrast to the hairlessness the pathogenesis regarding the lung emphysema is well described in the ITGB6-/mice. The condition is caused by an elevated expression of matrix metalloproteinase 12 (Mmp12), a macrophage expressed protease that degrades elastin causing a loss of lung elasticity and enlargement of alveolar airspaces. The elevated Mmp12 level, again, is caused by a lack of active transforming growth factor beta-1 (TGF- $\beta 1$ ), which has a regulatory effect on the expression of MMP12 [2]. It has been shown that integrin $\alpha_{v} \beta_{6}$ is central in activating TGF$\beta 1$. TGF- $\beta 1$ is secreted in an inactive form bound to a latency associated peptide (LAP), but binding of $\alpha_{\mathrm{v}} \beta_{6}$ to the LAP part of the LAP-TGF- $\beta 1$ complex, allows TGF- $\beta 1$ to interact with its receptor [20]. Morris et al. [2] report a 200-fold increase in the MMP12 expression in ITGB6 knockout mice compared to wild-type mice.

Our results have supported neither ITGB6 nor ITGAV as candidate genes for juvenile hairlessness and age dependent emphysema in pigs. However, the resemblance with the ITGB6-/- knockout phenotype in mice is striking and it is obvious to expect the causative mutation in the pig to affect a pathway analogous to the murine integrin $\alpha_{v} \beta_{6}-$ TGF- $\beta 1$ - Mmp12 - pathway. This pathway is, however, complex and elaborately regulated $[22,23]$ and involves several indispensable key elements in terms of maintaining full functionality. According to the database PigQTLdb [13] the region between S0149 and KS155 on pig chromosome 15 is syntenic to human chromosome 2 in the region from approximately $170 \mathrm{Mb}$ to $220 \mathrm{Mb}$. According to Ensembl release 49, March 2008, this region comprises 350 protein coding genes many of which are poorly annotated. Thus, it will be necessary to narrow down the candidate region further, before good predictions of candidate genes possibly involved in the biochemical pathway leading to the phenotype in question can be made. It is, however, also important to keep in mind that we can not yet completely rule out that the integrin $\alpha_{v} \beta_{6}$ is implicated in the phenotype since for instance a mutation in the promoter region or in a transcription factor/transcription factor target site could influence expression of either of the genes temporally or spatially. 
Table I: ITGB6 and ITGAV qPCR

\begin{tabular}{|c|c|c|c|c|c|c|}
\hline ITGB6 & & RPL4 & YWHAZ & HPRT & Ex9-10 & Ex3 \\
\hline & $E$ & 1.96 & 1.86 & 2.03 & 2.00 & 1.84 \\
\hline & SE & 0.11 & 0.00 & 0.00 & 0.25 & 0.24 \\
\hline \multirow[t]{2}{*}{ hh } & Mean & 18.31 & 20.37 & 22.99 & 26.17 & 27.16 \\
\hline & SE & 0.08 & 0.24 & 0.17 & 0.24 & 0.21 \\
\hline \multirow[t]{4}{*}{$\mathrm{HH}$} & Mean & 18.30 & 19.94 & 23.14 & 25.58 & 26.72 \\
\hline & SE & 0.12 & 0.14 & 0.16 & 0.16 & 0.18 \\
\hline & Expression ratio & & & & 1.42 & 1.23 \\
\hline & $\mathrm{P}$-value & & & & 0.073 & 0.078 \\
\hline \multirow[t]{3}{*}{ ITGAV } & & RPL4 & TBP & HPRT & ExI-3 & \\
\hline & $E$ & 1.98 & 1.99 & 2.01 & 2.01 & \\
\hline & SE & 0.14 & 0.00 & 0.00 & 0.00 & \\
\hline \multirow[t]{2}{*}{ hh } & Mean & 25.11 & 21.91 & 24.01 & 21.54 & \\
\hline & SE & 0.94 & 0.13 & 1.17 & 0.26 & \\
\hline \multirow[t]{4}{*}{$\mathrm{HH}$} & Mean & 24.82 & 21.58 & 23.14 & 20.78 & \\
\hline & SE & 1.04 & 0.28 & 0.51 & 0.28 & \\
\hline & Expression ratio & & & & 1.208 & \\
\hline & $\mathrm{p}$-value & & & & 0.740 & \\
\hline
\end{tabular}

qPCR data for the two ITGB6 target sequences Ex9-10 and Ex3 and the three reference genes RPL4, YWHAZ and HPRT and the ITGAV target sequence ExI-3 and the three reference genes RPL4, TBP and HPRT. The hh group serves as control group for the HH group. All three P-values $>$ 0.05 indicating not significantly different expression levels in the hh and $\mathrm{HH}$ groups.

\section{Conclusion}

A mutated phenotype has been detected in the pig. The phenotype is characterized by hairlessness until puberty, thin skin with few hair follicles and absence of musculi arrectores pili and development of localized areas of emphysema in the lungs from the age of puberty. Linkage studies have unequivocally pointed out the region harbouring the mutation responsible for the phenotype. However, sequencing and functional studies of the most obvious candidate genes, ITGB6 and ITGAV have so far not supported either of them as the causative gene behind juvenile hairlessness and age dependent lung emphysema in pigs. Future work will therefore be directed at narrowing down the candidate region through linkage analysis in an extended experimental family. We are in the process of breeding additional animals for this purpose. Once the candidate region spans only a few megabases, re-sequencing will be performed in $\mathrm{HH}$ and hh animals with the purpose of identifying the mutation explaining the phenotype in question. Further characterization of the mutated gene or locus and the effect of the mutation will provide novel information about the biochemical pathway involved in the development of lung emphysema.

\section{Methods}

Experimental population and DNA

Based on the founder boar an experimental population of 113 pigs was established and an EDTA stabilized blood sample was collected from each animal. DNA was extracted using a salt precipitation method [24].

\section{Histopathology}

From a two months old litter representing all three genotypes, three piglets of each phenotype were euthanized. Besides, 13 mature pigs (ages from eight to 42 months) were euthanized. Tissue samples from all lung lobes as well as skin samples from two locations on the lateral part of the left shoulder and thigh, respectively, were collected for histological evaluation. Following fixation for 24 hours in $10 \%$ neutral buffered formalin, the tissues were transformed to $70 \%$ ethanol for 48 hours. Tissues were processed through graded concentrations of ethanol and xylene, and embedded in paraffin blocks. The procedure of paraffin-embedment ensured that the 4-5 $\mu$ m thick tissue sections, which were stained with haematoxylin and eosin or trichrome [25], were cut perpendicular to the tissue surface.

All tissue sections were examined histologically using an Olympus BX60 microscope. Besides, the thickness of the dermis and epidermis was measured (mm) (18 observations pr. animal) and the number of hairs (encompassing hair roots and hair follicles) was counted (4 counts pr. animal) from randomly obtained standard microphotos of each skin section. A Leica DC500 camera was used for this purpose and for all histological pictures presented. The data were analyzed using the generalized linear procedure (GLM), SAS ${ }^{\circledast}$ System version 8.2 and a significance level of $\mathrm{p}<0.05$.

For immunohistochemistry, 3- to $4-\mu \mathrm{m}$ sections of paraffin-embedded lung tissue from seven pigs representing young and adult animals of the three genotypes (hh, $\mathrm{Hh}$ 
and $\mathrm{HH}$ ) were mounted on adhesive slides (Superfrost $\mathrm{R}$ Plus; Menzel-Glaser, Germany) and kept at $4{ }^{\circ} \mathrm{C}$ until processed.

For the primary antibody (polyclonal goat anti-mouse integrin $\alpha v \beta 6$ antibody) to work on porcine lung tissue, immunohistochemical stainings were initially performed with different procedures according to antigen retrieval methods and dilution of the primary antibody: a comparable positive reaction was obtained in sections of normal murine and normal porcine lung tissue when the concentration of primary antibody was $0.02 \mathrm{mg} / \mathrm{ml}$ and the antigen retrieval was performed with microwave oven boiling in Tris-EDTA buffer, $\mathrm{pH} 9$ for $2 \times 5 \mathrm{~min}$. Before the antigen retrieval, the sections were heated at $70^{\circ} \mathrm{C}$ for $15 \mathrm{~min}$. and then processed through xylene and rehydrated in graded concentrations of ethanol. After antigen retrieval and quenching of endogenous peroxidase activity $\left(0.6 \% \mathrm{H}_{2} \mathrm{O}_{2}\right.$ for $15 \mathrm{~min}$.) the sections were incubated with $5 \%$ normal rabbit serum (\#X0902 DakoCytomation Norden A/S, Glostrup, Denmark) in TBS for $30 \mathrm{~min}$. to reduce nonspecific positive reaction. The sections were incubated with the primary antibody (\#AF2389, R\&D Systems Minneapolis, MN, USA) in 5\% normal porcine serum in TBS, $\mathrm{pH}$ 7.6. for $18 \mathrm{~h}$ at $4^{\circ} \mathrm{C}$, followed by washes in TBS. A secondary antibody (rabbit anti-goat \#Z0454 DakoCytomation Norden A/S) was applied followed by wash in TBS. The sections were then incubated with goat peroxidase anti-peroxidase (\#B0157 DakoCytomation Norden A/S) and positive reaction was visualized using the chromogenic substrate diaminobenzidine (\#4170 Kem-En-Tec Diagnostics A/S, Taastrup, Denmark).

Counterstaining was performed for $10 \mathrm{~s}$ in Meyer's hematoxylin and washed $1 \mathrm{~min}$. in running tap water and 4 min. in distilled water. Finally, sections were mounted with glycerol-gelatin. Control immunostainings were run on parallel sections without the primary antibody and with a nonsense polyclonal IgG antibody of the same concentration as the primary antibody. Sections of murine lung tissue served as positive controls for specific staining of integrin $\beta 6$. The tissues were screened for reactivity following the optimal antigen retrieval procedure and dilution of the primary antibody.

\section{Linkage study}

Seven microsatellite markers: SW118, S0149, SW1945, SW2608, SW1983, SW906 and KS155 [15] covering the region from 60 to $100 \mathrm{cM}$ on SSC15 were selected and genotyped in the experimental family. Primers were labelled with fluorescent dyes and standard PCR were performed. Fragment analysis was done using an ABI (USA) PRISM $^{\circledast} 3100$ Genetic Analyzer and ABI GeneMapper ${ }^{\mathrm{rm}}$ Genotyping Software. For the linkage analysis the
TWOPOINT, ALL and CHROMPIC options in CRI-MAP 2.4 [26] were used.

\section{ITGB6 coding sequence}

Total RNA was isolated from lung tissue samples from two hh piglets using Qiagen (Germany) RNeasy Maxi kit according to the supplier's instructions. Four micrograms of the total RNA were incubated for $30 \mathrm{~min}$ at $37^{\circ} \mathrm{C}$ with RQ1 RNase-Free Dnase (Promega, USA) in a 20- $\mu$ l reaction. The samples were then phenol extracted and precipitated. The DNAse treated total RNA was mixed with $2 \mu \mathrm{g}$ of oligo $\mathrm{dT}_{15}$ primer and heated to $70^{\circ} \mathrm{C}$ for $5 \mathrm{~min}$, placed on ice and reverse transcribed into cDNA using $400 \mathrm{U} \mathrm{M}$ MLV Reverse Transcriptase and $50 \mathrm{U}$ of Rnasin ${ }^{\circledR}$ Ribonuclease Inhibitor (Promega). The reaction was incubated at $42^{\circ} \mathrm{C}$ for $1 \mathrm{~h}$ and terminated by heat inactivation at $95^{\circ} \mathrm{C}$ for $5 \mathrm{~min}$.

For primer design theITGB6 coding sequence from 5 species (Bos taurus (Genbank: NM_174698), Homo sapiens (Genbank: NM 000888), Mus musculus (Genbank: NM 021359) and Rattus norwegicus (Genbank: NM 001004263)) were aligned using "Multialin" [27]. Primers were designed from the consensus sequence in conserved regions that border a 1364 base pairs long central fragment of the ITGB 6 coding sequence. The fragment was amplified using Elongase ${ }^{\circledast}$ Enzyme Mix, Invitrogen ${ }^{\mathrm{TM}}$ (USA) and the primers ITGB6.2.F and ITGB6.2.R (see Table 2) according to the Elongase ${ }^{\circledast}$ Enzyme Mix, Invitrogen $^{\mathrm{TM}}$ protocol. The PCR products were run on a $1 \%$ agarose gel, appropriate bands were cut out and purified using GFX PCR DNA and Gel Purification Kit, Amersham Biosciences (UK).

The 5'- and 3'- ITGB6 specific sequence ends were amplified in the hh lung CDNA samples using BD SMART ${ }^{\text {TM }}$ RACE cDNA Amplification Kit, BD Biosciences (USA) and the gene specific primers, GSP1 and GSP2 (see Table 2) following the user's manual. The PCR products were evaluated on a $1.2 \%$ agarose gel. The 5 ' and 3' PCR products showed distinct bands of approximately 1 and $1.4 \mathrm{~kb}$ respectively. These bands were purified using GFX PCR DNA Purification Kit, Amersham Biosciences.

For cloning of the PCR products Zero Blunt ${ }^{\circledR} \mathrm{TOPO}^{\circledR} \mathrm{PCR}$ Cloning Kit for Sequencing (Invitrogen) was used according to the manufacturer's instructions.

Plasmids were isolated from 6 colonies using QIAprep ${ }^{\circledR}$ Spin Miniprep Kit (Qiagen, Germany). Inserts were PCR amplified using T3 and T7 primers.

BigDye ${ }^{\circledR}$ Terminator v3.1 Cycle Sequencing Kit, Applied Biosystems (USA), was used for the sequencing reaction with T3, T7, M13, ITGB6.2.F and ITGB6.2.R primers 
Table 2: Primer sequences

\begin{tabular}{|c|c|}
\hline Primer & Sequence \\
\hline ExonI5L & 5'-CAGAGGCTCTACCAGCACCT-3' \\
\hline ExonI5_R2 & 5'-TTCGGGAGTAAAGCAGTTCT-3' \\
\hline ITGB6.2.F & 5'-CCTACATTTGGATTCAAGCAC-3' \\
\hline ITGB6.2.R & 5'-CTCCGTTTAGAGTTACAGGG-3' \\
\hline GSPI & 5'-CCCGCCAGTTTGCTGTCCATTCCAA-3' \\
\hline GSP2 & 5'-GGGACTGTGTGTGCGGCAAGTGCAT-3 \\
\hline ITGB6_seq0IL & 5'-AGAACAAGTTCATCTGTATGAGAAT-3' \\
\hline ITGB6_seq0IR & 5'-AAATGTTTCCATAAGGAGACAA-3' \\
\hline Exonl+2_seqL & 5'-TCTTTACCTGTCCAGGTAGC-3' \\
\hline $\mathrm{E} \times 2 \mathrm{~L}$ & 5'-ATGGGGATTGAACTGCTTTG-3' \\
\hline Ex2R & 5'-GGAGAGAGGGTTTTCGATGA-3' \\
\hline Exonl0_R & 5'-TGCCTGCTTCTTTTCTCACA-3' \\
\hline Exon9+10_seqL & 5'-GTCTGAGGTGGAGCTGGAA-3' \\
\hline Exon $13 \mathrm{~L} 2$ & 5'-CGAAGGGAAAACCATCATTC-3' \\
\hline Exon3+4_seqL & 5'-TTTACTTATCTCTCTGGAGTTGGAGA-3' \\
\hline Exon9_II_L & 5'-CCCACACCAAAAGAAATGCT-3' \\
\hline MINF & 5'-AAAGAGCTGAAGGAAGGTTTGA-3' \\
\hline MINR & 5'-GCTTTTTCACCGAAAGCAAG-3' \\
\hline AVIF & 5'-CGACAGGCTCACATTCTACTTG-3' \\
\hline AVIR & 5'-TCAGTCTCAGGGTTCTCCTTGT-3' \\
\hline $\mathrm{AV} 2 \mathrm{~F}$ & 5'-GGAACAGCTCTCAAAGTTTCCT-3' \\
\hline $\mathrm{AV} 2 \mathrm{R}$ & 5'-GCACCTTCTCCTTGATTCTGAG-3' \\
\hline $\mathrm{AV} 3 \mathrm{~F}$ & 5'-AGGAGACTTCCAGACGACAAAG-3' \\
\hline AV3R & 5'-TCATGTTCTTGGAGTGACTTGG-3' \\
\hline AV7F & 5'-AGAGAGAGCCTGTTGGAACTTG-3' \\
\hline AV7R & 5'-CACTTCCСTTCAAGGATCTGAG-3' \\
\hline AV8F & 5'-GCTGTTTTAGCTGCTGTTGAGA-3' \\
\hline AV8R & 5'-TGTCCTCAATTGGAAGGTTCTT-3' \\
\hline AV9F & 5'-TCATTGAGGGAGATGTTCACAC-3' \\
\hline AV9R & 5'-ATGGGTCAGGATGTAGCGTAAC-3' \\
\hline SGF & 5'-ACTTCGGCGATGGCTTCT-3' \\
\hline SGR & 5'-CGGGTAGAAGACCAGTCACATT-3' \\
\hline INIIF & 5'-GATTTTCTGTCGCTGCCACT-3' \\
\hline INIIR & 5'-CAGCTTGGTCGTTTGGAAGT-3' \\
\hline$A V e x I-2 F$ & 5'-ATG CAA CAG GCA ACA GAG ACT-3 \\
\hline $\mathrm{AVex} 3 \mathrm{R}$ & 5'-TCC TGA AGA AAG CAA GTT CCA-3' \\
\hline
\end{tabular}

according to the manufacturer's protocol. Sequencing was done using ABI PRISM ${ }^{\circledast} 3100$ Genetic Analyzer. Based on the sequence information generated by analyzing these sequences, an additional set of sequencing primers, ITGB6_seq01L and ITGB6_seq01R (see Table 2) was designed for sequencing the central part of the ITGB6 coding sequence.

The generated trace files were base called and quality checked using PHRED [28], vector sequences were masked out using CROSS MATCH [29], repetitive sequences were masked using REPEATMASKER [30], sequences were assembled into contigs using PHRAP [29] and viewed using CONSED [31].

\section{Sequencing of ITGB6 coding sequence in $\mathrm{HH}$}

cDNA from two $\mathrm{HH}$ animals was generated as described for the two hh animals. The ITGB6 was PCR amplified using the primers Exon1+2_seqL and Exon15_R2 (see Table 2) and Phusion ${ }^{\mathrm{TM}}$ High-Fidelity DNA Polymerase (Finnzymes, Finland) according to the manufacturer's instructions. The PCR products were sequenced with these primers as well as Ex2L, Ex2R, Ex6R, Exon10R, Exon9+10_seqL and Exon13L2 (see Table 2) as previously described.

The HH sequences were assembled with the hh sequences as described earlier and the assembly was scrutinized for sequence differences between the two genotype groups.

\section{ITGAV coding sequence}

Seventeen porcine EST clones [32] with inserts homologous to the human ITGAV coding sequence [Genbank: NM 002210] were cultured overnight at $37^{\circ} \mathrm{C}$ on LB agar plates [33] containing $0.01 \%$ carbaniciline. One colony representing each clone were inoculated in LB broth $[33,33]$ containing $0.01 \%$ carbaniciline. Plasmids were isolated using QIAprep ${ }^{\circledR}$ Spin Miniprep Kit (Qiagen) according to the manufacturer's instructions. The purified plasmids were cut with the restriction enzymes XhoI and EcoRI at $37^{\circ} \mathrm{C}$ overnight and inserts were PCR amplified using T3/T7 or T3/2390R. PCR products were sequenced using BigDye ${ }^{\otimes}$ Terminator v3.1 Cycle Sequencing Kit (Applied Biosystems) and the same primers. Based on consensus sequences from the clones and the human ITGAV coding sequence, eight primer sets (AV primers, see Table 2) were designed to cover almost the entire porcine ITGAV coding sequence. Two porcine shotgun sequences (BL_75693 and BDE_82477) [34] aligning with the human ITGAV coding sequence were used to design the primer set (SG primers, see Table 2) for amplifying the $5^{\prime}$ end of the coding sequence. The nine ITGAV primer sets were used in standard PCR reactions in lung CDNAs from one hh and one HH pig. PCR products were sequenced using BigDye ${ }^{\circledast}$ Terminator v3.1 Cycle Sequencing Kit, (Applied Biosystems).

$\mathrm{HH}$ and hh sequences were assembled as described for the ITGB6 sequences and the assembly was scrutinized for sequence differences between the two genotype groups.

\section{RH mapping of ITGB6 and ITGAV}

The porcine ITGB6 and ITGAV were mapped in the IMpRH panel $[14,35,36]$. For ITGB6 a standard PCR reaction was applied with the primers: Exon15L (annealing in exon 15) and Exon15_R2 (annealing in the 3'UTR, see Table 2). For mapping of ITGAV, intron 11 was first PCR amplified and sequenced as described previously using the primers IN11F and IN11R (see Table 2) annealing in exon 11 and 12 respectively. RH mapping was performed with the primers MINF and MINR (both annealing in intron 11, see Table 2). PCR amplification in the 118 
hybrids was evaluated after electrophoresis on a 1.2\% agarose gel.

\section{Quantitative PCR}

Lung RNA samples from each of four HH pigs (from eight to 22 months old) and four hh pigs from another farm (eight months old) were generated using TRI REAGENT ${ }^{\oplus}$, Molecular Research Center (Ohio) according to the manufacturer's instructions. RNA samples were DNAse treated using RNeasy ${ }^{\circledR}$ Mini Kit (Qiagen). cDNAs were synthesized as described earlier.

For ITGB6 the cDNA was diluted five fold. Two primer sets were used: one within exon 3 (Exon3+4_seqL and Ex2R, see Table 2) and one spanning the exon 9/exon 10 boundary (Exon9_11_L and Exon10_R, see Table 2).Ribosomal protein L4 (RPL4), hypoxanthine phosphoribosyl-transferase (HPRT) and tyrosine 3-monooxygenase/tryptophan 5-monooxygenase activation protein, zeta-polypeptide (YWHAZ) were included in the study as reference genes.

For ITGAV the CDNA was undiluted and the primers AVex2F (annealing at the exon1-exon2 boundary) and AVex3R (annealing in exon 3) (see Table 2) were used. The reference genes were Ribosomal protein L4 (RPL4), hypoxanthine phosphoribosyl-transferase (HPRT) and TATA binding protein $(T B P)$.

An MX3000 $\mathrm{p}^{\mathrm{TM}}$ thermocycler (Stratagene, USA) was used for the real time quantitative PCR. For each primer set a standard curve was constructed using a dilution series of known concentrations of purified PCR products (QIAquick $^{\circledast}$ PCR Purification Kit (250), Qiagen). The standard curves represented a plot of CP (crossing point, defined as the cycle at which the fluorescence signal crosses the threshold) vs. the relative concentration of PCR product. Single PCR reactions were performed for each cDNA sample, a genomic DNA sample and a nontemplate negative control using Brilliant ${ }^{\circledast}$ SYBR $^{\circledast}$ Green Master Mix (Stratagene). SYBR green fluorescence was automatically detected during each PCR reaction. The MX3000 ${ }^{\mathrm{TM}}$ software (Stratagene) was used for baseline adjustment and accordingly for setting the fluorescence threshold value. CP values for the HH group obtained from all three target sequences was compared to $\mathrm{CP}$ values for the hh "control" group using REST [18] with 2000 randomisations. A significance level of $\mathrm{p}<0.05$ was chosen.

\section{Western Blot}

Protein was isolated from lung tissue samples from mouse, one hh pig, one Hh pig and one HH pig using TRI REAGENT ${ }^{\circledast}$, Molecular Research Center (Ohio) following the manufacturers instructions. Eighty $\mu \mathrm{g}$ of each protein sample as well as ten $\mu$ l of the marker SeeBlue Plus2 (Invitrogen) were loaded on a NuPAGE ${ }^{\mathrm{TM}} 4-12 \%$ Bis-Tris gel and run for 55 minutes at $200 \mathrm{~V}$ using the XCell SureLock ${ }^{\mathrm{TM}}$ Mini-Cell, Invitrogen. The proteins were blotted onto a PVDF membrane, Invitrogen, using an XCell $\mathrm{II}^{\mathrm{TM}}$ Blot Module, Invitrogen, according to the manufacturer's protocol. The membrane was stained with Ponceau S, Sigma (USA) to verify successful transfer of proteins. Detection was performed using WesternBreeze ${ }^{\circledast}$ Chemilunimescent Western Blot Immunodetection Kit, Invitrogen and the antibody Anti-mouse Integrin $\beta 6$ Antibody (\#AF2389, $\mathrm{R} \& \mathrm{D}$ Systems Minneapolis, MN, USA). A concentration of $0.4 \mu \mathrm{g}$ antibody per ml primary antibody diluent was used. A BioMax Light film, Kodak, was exposed for 10 minutes.

\section{Flow cytometry analysis}

An indirect three-step immuno-fluorescence staining method for porcine B-cells [37] was adapted to label trypsinised primary monolayer kidney cells from the 4 pigs ( $2 \mathrm{HH}$ and $2 \mathrm{hh}$ ). A volume of $100 \mu \mathrm{l}$ cell suspension $\left(10 \times 10^{6}\right.$ cells $\left./ \mathrm{ml}\right)$ was incubated with $25 \mu \mathrm{l}(10 \mu \mathrm{g} / \mathrm{ml})$ of the anti- integrin $\alpha_{\mathrm{v}} \beta_{6}$ mAb MAB2077Z (Chemicon International Inc, Denmark). After washing with $2 \mathrm{ml}$ PBS supplemented with $0.1 \%$ sodium azide (washing buffer), the cells were incubated for 30 min with $25 \mu \mathrm{l}$ (1:100 dilution) biotin-conjugated goat-anti-mouse (BIOGAM) IgG (Jackson Immuno Research Laboratories (JIRL) Inc., West Grove, PA, USA) diluted in washing buffer. After another washing, the cells were incubated with $25 \mu \mathrm{l}$ (1:50 dilution) R-phycoerythrin (RPE)-conjugated streptavidin (R0438, DAKO, Denmark) for 10 min. After washing, the cells were finally resuspended in $200 \mu \mathrm{l}$ BD FACSFlow ${ }^{\mathrm{TM}}$ (Becton Dickinson) for flow cytometric examination. All incubations were performed at room temperature in the dark and centrifugations were carried out at $490 \times g$ at $24^{\circ} \mathrm{C}$. Controls without any reagents and with secondary antibody, only, were included for each pig. In each test, 10,000 cells were counted using a FACScan flow cytometer (Becton Dickinson) for data acquisition, and data were analysed using the Cell-Quest ${ }^{\mathrm{TM}}$ software (Becton Dickinson).

\section{Authors' contributions}

CSB carried out the main part of the genetics laboratory work, the sequence and linkage analysis, tissue sampling and drafted the paper. CBJ assisted in the sequence and linkage analysis. LB carried out the ITGAV sequencing, RH mapping and qPCR. SC participated in the RNA extraction, qPCR analysis and the western blotting. HEJ and PSL carried out the histological and immunohistochemical studies. JN carried out the flow cytometry analysis. KC established the experimental population and carried out the SAS analysis. MF initiated the study, carried out the comparative mapping, blood and tissue sampling and coordinated the work. All authors contributed to and approved the final manuscript. 


\section{Acknowledgements}

We thank Tina Neergaard Mahler, Majken Madvig Jansen, Signe Anker Madsen, Minna Jacobsen and Lennart Engberg Carlsen for their excellent technical assistance. We thank Ann-Britt Nygård for help and advices for the quantitative $P C R$ procedure.

This work was funded by a grant from the Danish Agricultural and Veterinary Research Council and a Ph.D. stipend from The Royal Veterinary and Agricultural University, Denmark to Camilla Sichlau Bruun.

\section{References}

I. Huang XZ, Wu JF, Cass D, Erle DJ, Corry D, Young SG, et al:: Inactivation of the integrin beta 6 subunit gene reveals a role of epithelial integrins in regulating inflammation in the lung and skin. J Cell Biol 1996, I33:921-928.

2. Morris DG, Huang X, Kaminski N, Wang Y, Shapiro SD, Dolganov G, et al.: Loss of integrin alpha(v)beta6-mediated TGF-beta activation causes Mmp I2-dependent emphysema. Nature 2003, 422:169-173.

3. Hynes RO: Integrins: versatility, modulation, and signaling in cell adhesion. Cell 1992, 69: I |-25.

4. Busk M, Pytela R, Sheppard D: Characterization of the integrin alpha $\mathbf{v}$ beta 6 as a fibronectin-binding protein. I Biol Chem 1992, 267:5790-5796.

5. Sheppard D: Functions of pulmonary epithelial integrins: from development to disease. Physiol Rev 2003, 83:673-686.

6. Bader BL, Rayburn H, Crowley D, Hynes RO: Extensive vasculogenesis, angiogenesis, and organogenesis precede lethality in mice lacking all alpha $\mathbf{v}$ integrins. Cell 1998, 95:507-5I9.

7. Breuss JM, Gallo J, DeLisser HM, Klimanskaya IV, Folkesson HG, Pittet JF, et al.: Expression of the beta 6 integrin subunit in development, neoplasia and tissue repair suggests a role in epithelial remodeling. J Cell Sci I995, I 08(Pt 6):224I-225I.

8. Arend LJ, Smart AM, Briggs JP: Mouse beta(6) integrin sequence, pattern of expression, and role in kidney development. J $\mathrm{Am}$ Soc Nephrol 2000, I I:2297-2305.

9. Agrez M, Chen A, Cone RI, Pytela R, Sheppard D: The alpha v beta 6 integrin promotes proliferation of colon carcinoma cells through a unique region of the beta 6 cytoplasmic domain. J Cell Biol 1994, I 27:547-556.

10. Haapasalmi K, Zhang K, Tonnesen M, Olerud J, Sheppard D, Salo T, et al.: Keratinocytes in human wounds express alpha $v$ beta 6 integrin. J Invest Dermatol 1996, 1 06:42-48.

I I. NCBI Map Viewer [http://www.ncbi.nlm.nih.gov/mapview/]

12. Mouse Genome Informatics [http://www.informatics.jax.org]

13. Hu ZL, Reecy JM: Animal QTLdb: beyond a repository. A public platform for QTL comparisons and integration with diverse types of structural genomic information. Mamm Genome 2007, I 8: 1-4.

14. Meyers SN, Rogatcheva MB, Larkin DM, Yerle M, Milan D, Hawken RJ, et al.: Piggy-BACing the human genome II. A high-resolution, physically anchored, comparative map of the porcine autosomes. Genomics 2005, 86:739-752.

15. U.S. Meat Animal Research Center [http:// www.marc.usda.gov/]

16. Sheppard D, Rozzo C, Starr L, Quaranta V, Erle DJ, Pytela R: Complete Amino-Acid-Sequence of A Novel Integrin Beta-Subunit (Beta-6) Identified in Epithelial-Cells Using the Polymerase Chain-Reaction. Journal of Biological Chemistry 1990, 265: II 502-II507.

17. de Melker AA, Sonnenberg A: Integrins: alternative splicing as a mechanism to regulate ligand binding and integrin signaling events. Bioessays 1999, 21:499-509.

18. Pfaffl MW, Horgan GW, Dempfle L: Relative expression software tool (REST) for group-wise comparison and statistical analysis of relative expression results in real-time PCR. Nucleic Acids Res 2002, 30:e36.

19. Jorsal SE, Bille-Hansen V, Vigre H, Larsen PB, Bøtner A, Nielsen EO, et al.: PMWS-laboratory diagnosis on herd and pig level in a Danish case-control study. Proceedings of the 19th IPVS Cngress, Copenhagen, Denmark -270.

20. Munger JS, Huang X, Kawakatsu H, Griffiths MJ, Dalton SL, Wu J, et al.: The integrin alpha $v$ beta 6 binds and activates latent TGF beta I: a mechanism for regulating pulmonary inflammation and fibrosis. Cell 1999, 96:319-328.

21. Bader BL, Rayburn H, Crowley D, Hynes RO: Extensive vasculogenesis, angiogenesis, and organogenesis precede lethality in mice lacking all alpha v integrins. Cell 1998, 95:507-5 I9.

22. Jenkins RG, Su X, Su G, Scotton CJ, Camerer E, Laurent G], et al.: Ligation of protease-activated receptor I enhances alpha(v)beta6 integrin-dependent TGF-beta activation and promotes acute lung injury. J Clin Invest 2006, I | 6: |606-|6 |4.

23. Chapman HA: Disorders of lung matrix remodeling. J Clin Invest 2004, I I 3: |48- I 57.

24. Grimberg J, Nawoschik S, Belluscio L, McKee R, Turck A, Eisenberg $A$ : A simple and efficient non-organic procedure for the isolation of genomic DNA from blood. Nucleic Acids Res 1989 , I 7:8390.

25. Manual of Histologic Staining Methods of the Armed Forces Institute of Pathology 3rd edition. McGraw-Hill, New York; 1960.

26. Green P, Falls K, Crooks S: Documentation for CRI-MAP, Version 2.4 Washington University School of Medicine, St. Louis; 1990.

27. Corpet F: Multiple sequence alignment with hierarchical clustering. Nucleic Acids Res 1988, 16:10881-10890.

28. Ewing B, Hillier L, WendI MC, Green P: Base-calling of automated sequencer traces using phred. I. Accuracy assessment. Genome Res 1998, 8:175-185.

29. Laboratory of Phil Green, Genome Sciences Department, University of Washington [http://www.phrap.org]

30. Institute for Systems Biology [http://www.repeatmasker.org/]

31. Gordon D, Abajian C, Green P: Consed: a graphical tool for sequence finishing. Genome Res 1998, 8:195-202.

32. Gorodkin J, Cirera S, Hedegaard J, Gilchrist MJ, Panitz F, Jorgensen C, et al:: Porcine transcriptome analysis based on 97 non-normalized cDNA libraries and assembly of I,02 I,89I expressed sequence tags. Genome Biol 2007, 8:R45.

33. Sambrook J, Fritsch EF, Maniatis T: Molecular Cloning : a laboratory manual 2 nd edition. 1989.

34. Wernersson R, Schierup MH, Jorgensen FG, Gorodkin J, Panitz F, Staerfeldt $\mathrm{HH}$, et al:: Pigs in sequence space: a $0.66 \mathrm{X}$ coverage pig genome survey based on shotgun sequencing. $B M C$ Genomics 2005, 6:70.

35. Milan D, Hawken R, Cabau C, Leroux S, Genet C, Lahbib Y, et al.: IMpRH Server: an RH mapping server available on the Web. Bioinformatics 2000, I 6:558-559.

36. RH mapping on INRA-University of Minnesota porcine Radiation Hybrid panel [http://rhdev.toulouse.inra.fr]

37. Nielsen J, Vincent IE, Botner A, Ladekaer-Mikkelsen AS, Allan G, Summerfield A, et al: Association of lymphopenia with porcine circovirus type 2 induced postweaning multisystemic wasting syndrome (PMWS). Vet Immunol Immunopathol 2003, 92:97- I I I.

Publish with Bio Med Central and every scientist can read your work free of charge

"BioMed Central will be the most significant development for disseminating the results of biomedical research in our lifetime. "

Sir Paul Nurse, Cancer Research UK

Your research papers will be:

- available free of charge to the entire biomedical community

- peer reviewed and published immediately upon acceptance

- cited in PubMed and archived on PubMed Central

- yours - you keep the copyright 